\section{Depression in Japanese Patients With COPD: A Cross-Sectional Study}

\section{To the editor:}

I read the study by Horita et al "Depression in Japanese Patients With COPD: A Cross-Sectional Study," ${ }^{1}$ in which the severity and prevalence of depression were found to be associated with the severity and other parameters of COPD. ${ }^{1}$

In addition to the COPD parameters, obstructive sleep apnea (OSA) should be added as a risk factor for depression. The comorbid association of COPD and OSA is called the "overlap syndrome."2 Chen et al evaluated 2,818 patients diagnosed with OSA, and 14,090 matched non-OSA enrollees used as a comparison cohort. ${ }^{3}$ The reported incidence of depression per thousand person-years was about twice as high among patients with OSA as those without OSA. ${ }^{3}$ Furthermore, Best et al studied 82 outpatients with treatment-resistant depression who underwent overnight polysomnography, and found that many individuals with treatment-resistant depression have OSA. ${ }^{4}$

The effect of CPAP treatment on depression was evaluated by Diamanti et al, in 24 patients with depression and OSA, who were followed for at least 6 months after initiation of CPAP. ${ }^{5}$ CPAP improved quality of life and decreased depression symptoms. ${ }^{5}$ In a similar study, El-Sherbini et al explored the effect of CPAP in 37 patients with depression and OSA, before and 2 months after initiating CPAP. ${ }^{6}$ Their findings suggested that patients with OSA should be screened for depression, and that CPAP should be tried first, before other depression therapies. ${ }^{6}$
COPD, overlap syndrome, and depression are intertwined. When evaluating depression in patients with COPD, screening for OSA should be performed, and CPAP treatment, if indicated, should follow. This approach may improve the control of depression in patients with COPD.

Samer Alkhuja MD

Commonwealth Medical College Pocono Medical Center

East Stroudsburg, Pennsylvania

The author has disclosed no conflicts of interest.

DOI: $10.4187 /$ respcare. 02855

\section{REFERENCES}

1. Horita N, Kaneko T, Shinkai M, Yomota M, Morita S, Rubin BK, Ishigatsubo Y. Depression in Japanese patients with chronic obstructive pulmonary disease: a cross-sectional study. Respir Care 2013; 58(7):1196-1203.

2. Lee R, McNicholas WT. Obstructive sleep apnea in chronic obstructive pulmonary disease patients. Curr Opin Pulm Med 2011; 17(2):79-83

3. Chen YH, Keller JK, Kang JH, Hsieh HJ, Lin HC. Obstructive sleep apnea and the subsequent risk of depressive disorder: a population based follow-up study. J Clin Sleep Med 2013;9(5):417-423.

4. Best MW, Fitzpatrick M, Milev R, Bowie $\mathrm{CR}$, Jokic R. Utility of the Berlin questionnaire for predicting obstructive sleep apnea in individuals with treatment-resistant depression. Sleep Breath 2013 [Epub ahead of print].

5. Diamanti C, Manali E, Ginieri-Coccossis M, Vougas K, Cholidou K, Markozannes E, et al. Depression, physical activity, energy consumption, and quality of life in OSA patients before and after CPAP treat- ment. Sleep Breath 2013 [Epub ahead of print].

6. El-Sherbini AM, Bediwy AS, El-Mitwalli A. Association between obstructive sleep apnea and depression and the effect of continuous positive airway pressure treatment. Neuropsychiatry Dis Treat 2011;7(1):715721.

\section{Depression in Japanese Patients With COPD: A Cross-Sectional Study-Reply}

In Reply:

Alkhuja and colleagues remind us that CPAP treatment may be effective for treating depression seen in patients with COPD and obstructive sleep apnea (OSA): the overlap syndrome. These conditions are interrelated in that COPD and OSA are inflammatory conditions; depression can increase smoking, decrease medication adherence, and cause sleep disturbance; and poor sleep quality can worsen depression. Although CPAP treatment is not used for patients who have COPD alone, in persons with both COPD and depression it seems worthwhile to screen for OSA with a sleep study, as unrecognized OSA may contribute to the severity of the other conditions.

Nobuyuki Horita MD on behalf of the authors

Department of Internal Medicine and Clinical Immunology

Yokohama City University Graduate School of Medicine Yokohama, Japan

The authors have disclosed no conflicts of interest.

DOI: $10.4187 /$ respcare. 02890 